\title{
A special prognostic indicator: tumor mutation burden combined with immune infiltrates in lung adenocarcinoma with TP53 mutation
}

\author{
Jing $\mathrm{Fu}^{1 \#}$, Yaonan $\mathrm{Li}^{2 \#}$, Cuidan $\mathrm{Li}^{3}$, Yuyang Tong ${ }^{1}$, Mengyuan $\mathrm{Li}^{1}$, Shundong Cang ${ }^{1}$ \\ ${ }^{1}$ Department of Oncology, Henan Provincial People's Hospital, People's Hospital of Zhengzhou University, People's Hospital of Henan University, \\ Zhengzhou, China; ${ }^{2}$ Department of Emergency, Henan Provincial People's Hospital, People's Hospital of Zhengzhou University, People's Hospital \\ of Henan University, Zhengzhou, China; ${ }^{3}$ CAS Key Laboratory of Genome Sciences \& Information, Beijing Institute of Genomics, Chinese \\ Academy of Sciences, China National Center for Bioinformation, Beijing, China \\ Contributions: (I) Conception and design: J Fu, C Li; (II) Administrative support: S Cang; (III) Provision of study materials or patients: None; (IV) \\ Collection and assembly of data: J Fu, Y Li; (V) Data analysis and interpretation: J Fu, Y Li, C Li; (VI) Manuscript writing: All authors; (VII) Final \\ approval of manuscript: All authors. \\ "These authors contributed equally to this work. \\ Correspondence to: Prof. Shundong Cang. Department of Oncology, Henan Provincial People's Hospital, People's Hospital of Zhengzhou University, \\ People’s Hospital of Henan University, Zhengzhou, China. Email: cangshundong@163.com.
}

Background: TP53 mutation (TP5 $3^{\text {mut }}$ ) is significantly associated with immunotherapy response in lung adenocarcinoma (LUAD), but not an ideal independent prognostic predictor for it. Here, we investigated a novel potential biomarker and constructed a model for prognostic prediction in LUAD TP $53^{\text {mut }}$ patients.

Methods: 469 LUAD samples retrieved from The Cancer Genome Atlas database were divided into TP5 $3^{\mathrm{wt}}$ (wild-type TP53) and TP5 $3^{\text {mut }}$ groups. TMB values were calculated based on the number of variants/exon lengths, and high- and low-TMB groups were divided by the median value. Differentially expressed genes (DEGs) between the two TMB groups were identified using "limma" package, and functional analyses were performed by Kyoto Encyclopedia of Genes and Genomes, Gene Ontology, and Gene Set Enrichment Analysis. The infiltration ratio of 22 immune cells were calculated with the CIBERSORT algorithm. Survival analyses were estimated by Kaplan-Meier with the log-rank test. Finally a TMB prognostic index (TMBPI) with receiver operating characteristic (ROC) curve was constructed and calculated to evaluate the predictive value in TP5 $3^{\text {mut }}$ LUAD.

Results: There were diverse mutation types in 100\% of TP53 mutants, while mutations were present in $86.5 \%$ of cases with $T P 53^{\mathrm{wt}}$. TP $53^{\mathrm{mut}}$ patients had higher TMB levels than $T P 53^{\mathrm{wt}}$ patients. Overall survival in TP $53^{\text {mut }}$ patients with low-TMB levels was significantly shorter than that in high-TMB TP $53^{\text {mut }}$ patients. High-TMB patients had higher levels of CD8 T cell and effector B cell, while lower levels of resting memory CD4 T cells, monocytes, activated dendritic cells, etc. than low-TMB patients. Poor survival outcome in $T P 53^{\text {mut }}$ patients was correlated with lower effector B cell infiltration and higher activated dendritic cell. Survival risk analyses of 121 DEGs showed that good survival outcomes correlated positively with FBXO36 and KLHL35 expression levels, but correlated negatively with that of LINC0054. TMBPI analysis of the $T P 53^{\text {mut }}$ patients showed that high-TMBPI patients had worse survival outcomes than low-TMBPI patients.

Conclusions: Our findings suggest that the TMB value with immune infiltrates is a novel potential biomarker for prognostic prediction of TP $53^{\text {mut }}$ patients. The TMBPI combined with detection of TP53 mutation can be used as a better predictor of prognosis in LUAD.

Keywords: Lung adenocarcinoma (LUAD); TP53 mutation; tumor mutation burden; immune infiltrates; prognosis

Submitted Mar 30, 2021. Accepted for publication Jul 30, 2021.

doi: $10.21037 /$ tcr-21-565

View this article at: https://dx.doi.org/10.21037/tcr-21-565 


\section{Introduction}

Lung adenocarcinoma (LUAD) is the most common histological subtype of non-small cell lung carcinoma (NSCLC), accounting for more than $60 \%$ of NSCLC (1). LUAD is a leading cause of cancer-related mortality worldwide, with an average 5-year survival rate of only $15 \%$ (2). The TP53 gene has been known as a tumor suppressor since the 1990s (3). Somatic mutations in the TP53 gene occur in more than $50 \%$ of all LUAD and are the most frequent mutated alterations (4). TP53 mutation was reported that not only promotes tumor progression, but also is significantly associated with immunotherapy response (5-7). In 2015, the US Food and Drug Administration (FDA) approved immune checkpoint blockade (ICB) targeting programmed death-1 (PD-1)/programmed cell death ligand 1 (PD-L1) and cytotoxic T-lymphocyte-associated protein 4 (CTLA-4)/B7-1 for treating advanced LUAD (8). In recent years, immunotherapy have showed significant improvement in survival outcome of LUAD, where $T P 53^{\text {mut }}$ patients have better response to immunotherapy than $T P 53^{\mathrm{wt}}$ patients $(6,9,10)$. Consequently, many current studies consider TP $53^{\text {mut }}$ an independent predictor of immunotherapy response (6). Nonetheless, not all TP $53^{\text {mut }}$ patients can benefit from immunotherapy, and the objective response rate (ORR) and disease control rate (DCR) for $T P 53^{\text {mut }}$ patients to ICB immunotherapy were $47.4 \%$ and $57.9 \%$ respectively (11). Facing nearly $50 \%$ of $T P 53^{\text {mut }}$ patients with the low or no response to immunotherapy, we believe that $T P 53^{\text {mut }}$ is far from an ideal independent predictor thereof $(11,12)$. Therefore, there is an urgent need for exploration of novel potential immune-related biomarkers combined with detection of $T P 53^{\text {mut }}$ for precise prediction of the prognosis of LUAD.

To date, TP $53^{\text {mut }}$ related and non-related immune prognostic signatures for the prediction of overall survival and therapeutic responses in lung cancer mainly include PD-1/PD-L1 expression levels (13), microsatellite instability (14), tumor mutation burden (TMB) (15), neoantigen load (16), and tumor-infiltrating lymphocytes (TILs) (17). TMB is defined as the total number of nonsynonymous mutations per coding area of a tumor genome, and is calculated as mutations per megabase. Many studies have demonstrated that tumors with higher TMB tend to form more neoantigens to pose higher immunogenicity, and TMB has recently been identified as a genetic signature associated with favorable outcome for immunotherapy in many types of cancer (18). Furthermore, a series of KEYNOTE oncology clinical trials have implied that patients with NSCLC and PD-L1 positive expression [tumor proportion score (TPS) $\geq 1 \%$ ] benefited from pembrolizumab immunotherapy; in particular, patients with PD-L1 TPS of $\geq 50 \%$ had better survival outcome $(19,20)$. In addition, tumors can be classified as "cold" or "hot" based on the abundance of TILs in the tumor immune microenvironment (TIME) (21). In lung cancer, hot tumors demonstrate high response rates to immunotherapy, and converting a cold tumor to a hot tumor could confer more benefits to immunotherapy patients (22).

As a tumor suppressor, mutations in the TP53 gene can lead to 553 losing its regulatory roles in DNA repair, which may cause the higher TMB levels in TP $53^{\text {mut }}$ tumors $(23,24)$. Moreover, a higher expression level of PD-L1 in TP $53^{\text {mut }}$ patients was also identified $(25,26)$. Beyond that, as a series of clinical trials reported that PD-L1-negative or low-TMB patients also respond to immunotherapy (27), suggesting that PD-1/PD-L1 expression and TMB values are not the ideal biomarkers for it, and these may be attributed to other influencing factors in the TIME. Considering the limitation of single biomarker, a prognostic predictive model covering various biomarkers may direct immunotherapy more precisely. In addition, the correlation of these current biomarkers with survival outcome and immunotherapeutic responses in LUAD with TP $53^{\text {mut }}$ remain unclear. Here, we obtained the multi-omics data of patients with LUAD from The Cancer Genome Atlas (TCGA) database. Through comparison analysis, we investigated the relationship of PD-1/PD-L1 expression, TMB levels, and their potential association with immune infiltrates with survival outcome in LUAD patients with $T P 53^{\text {mut }}$ to identify a novel potential biomarker for prognostic prediction of TP $53^{\text {mut }}$ patients, and finally attempted to construct a prognostic predictive model for immunotherapy.

We present the following article in accordance with the REMARK reporting checklist (available at https://dx.doi. org/10.21037/tcr-21-565).

\section{Methods}

\section{Acquisition and processing of multi-omics data}

The study was conducted in accordance with the Declaration of Helsinki (as revised in 2013). First, we retrieved the multi-omics data of 469 LUAD samples from TCGA database by the GDC tool (http://portal. gdc.cancer.gov/). We identified 227 samples with $T P 53^{\text {mut }}$ and 242 samples with TP5 $3^{\text {wt }}$. Masked Somatic Mutation data of each sample was selected and processed 
through VarScan software. Somatic variants in Mutation Annotation Format (MAF) were prepared and analyzed with the $\mathrm{R}$ maftools package, which has multiple analysis modules for summarizing, analyzing, and visualizing MAF files (28). Meanwhile, the transcriptome profiles of all available TP $53^{\text {mut }}$ samples were downloaded. The corresponding clinical data of samples with and without $T P 53^{\text {mut }}$ were also obtained via the GDC tool, and included the variables age (years), sex (female and male), $\mathrm{T}$ (tumor size), $\mathrm{N}$ (metastatic lymph node), $\mathrm{M}$ (distant metastasis), American Joint Committee on Cancer tumor-nodemetastasis (AJCC TNM) stage (I-IV stage), and survival outcome mainly including overall survival time (OS).

\section{Precise calculation of TMB values}

TMB defined as the number of somatic mutations in the coding region per megabase, was calculated with the number of variants/exon lengths for each sample through Perl scripts based on the JAVA platform in our study. Then, we divided the samples into high- and low-TMB groups based on the median value.

\section{$R N A$ sequencing differential expression and patbway analysis}

According to the TMB levels, we classified the transcriptome data of TP $53^{\text {mut }}$ LUAD samples into high- and low-TMB groups. The differentially expressed genes (DEGs) between the two groups were identified using the limma package, with absolute $\log$ fold-change $(\log 2 \mathrm{FC})>1$ and false discovery rate $(\mathrm{FDR})<0.05$. A heatmap plot was drawn to exhibit the expression difference via the heatmap package. Then, org. Hs.eg.db: Genome wide annotation for Human was applied to obtain the Entrez Gene ID for each DEG, and we also performed function and pathway analyses using Gene Ontology (GO), Kyoto Encyclopedia of Genes and Genomes (KEGG). Further, Gene Set Enrichment Analysis (GSEA) was performed based on the JAVA platform with TMB levels as the phenotype and with c2.cp.kegg.v7.0 symbols.gmt gene sets as the reference gene set.

\section{CIBERSORT evaluation and prognostic analysis of immune cells in TP5 $3^{\text {mut }}$ LUAD}

The infiltration ratio of 22 immune cells from each sample were calculated with the CIBERSORT algorithm (R script v1.03) to evaluate the abundances of member cell types using gene expression data. The distributions of the immune cells in the two TMB groups were visualized via the pheatmap package. Moreover, univariate Cox analysis of 22 immune cells was conducted, fitted by the function coxph in the survival package.

\section{Survival analysis of DEGs and TIMER database}

We selected all DEGs with $|\log 2 \mathrm{FC}|>1$ and FDR $<0.05$ to assess their prognostic value in $T P 53^{\text {mut }}$ patients. We utilized the for cycle R script to perform batch survival analysis of the genes via the survival package, and association with survival outcome. Meanwhile, correlation of gene expression and copy number variations (CNVs) with immune infiltrates in LUAD was evaluated based on the gene and SCNA modules of the TIMER database (http:// cistrome.shinyapps.io/timer/) (29).

\section{Construction of TMB prognostic index (TMBPI) for bub genes}

We constructed a prognostic predictive model and obtained the respective coefficients $(\beta \mathrm{i})$ of three hub genes by performing multivariate Cox regression analysis. As previous reports (30), the TMBPI was defined as: TMBPI $=\Sigma(\beta \mathrm{i} \times \mathrm{Expi})(\mathrm{i}=3)$, and high- and low-risk groups were divided with reference to the median TMBPI as the threshold. Furthermore, Kaplan-Meier survival analysis was conducted between the two groups and the receiver operating characteristic (ROC) curve with area under the curve (AUC) was constructed and calculated to evaluate the predictive value of three signatures in TP $53^{\text {mut }}$ LUAD.

\section{Statistical analysis}

All statistical analyses of the present study were performed using R statistical software (version 4.0.3). The comparisons between two groups was tested by Wilcoxon rank-sum; and the Kruskal-Wallis test was used for $\geq 2$ categories. KaplanMeier survival analysis was examined by the log-rank test. Multivariate analysis was conducted via Cox regression. $\mathrm{P}<0.05$ was considered statistically significant.

\section{Results}

\section{Patient characteristics}

As the workflow in Figure S1, we collected the data of 469 patients with LUAD; TP5 $3^{\text {mut }}$ was identified in 227 cases 
Table 1 Baseline characteristics of 469 patients with LUAD with and without TP5 $3^{\text {mut }}$ from TCGA cohort

\begin{tabular}{|c|c|c|}
\hline Characteristics & TP53 ${ }^{\text {mut }}$ & $\mathrm{TP} 53^{\mathrm{wt}}$ \\
\hline \multicolumn{3}{|l|}{ Vital status } \\
\hline Alive & $137(60.35)$ & 159 (65.70) \\
\hline Dead & 90 (39.65) & $83(34.30)$ \\
\hline Age, y & $65.4 \pm 10.20$ & $66.68 \pm 9.62$ \\
\hline$\geq 65$ & $108(45.58)$ & $147(60.74)$ \\
\hline$<65$ & $113(49.78)$ & $82(33.88)$ \\
\hline Unknow & $6(2.64)$ & $13(5.37)$ \\
\hline \multicolumn{3}{|l|}{ Gender } \\
\hline Female & $122(53.74)$ & 133 (54.96) \\
\hline Male & $105(46.26)$ & $109(45.04)$ \\
\hline \multicolumn{3}{|l|}{ AJCC-T } \\
\hline T0 & 0 & 0 \\
\hline $\mathrm{T} 1-2$ & $198(87.22)$ & 210 (86.78) \\
\hline T3-4 & 27 (11.89) & $32(13.22)$ \\
\hline Unknow & $2(0.89)$ & $0(0.00)$ \\
\hline \multicolumn{3}{|l|}{ AJCC-N } \\
\hline NO & $141(62.11)$ & 158 (65.29) \\
\hline $\mathrm{N} 1$ & 48 (21.15) & 38 (15.70) \\
\hline $\mathrm{N} 2$ & $32(14.10)$ & 35 (14.46) \\
\hline N3 & $2(0.88)$ & $3(1.24)$ \\
\hline NX & $4(1.76)$ & $8(3.31)$ \\
\hline \multicolumn{3}{|l|}{ AJCC-M } \\
\hline MO & $147(64.76)$ & $173(71.49)$ \\
\hline M1 & $13(5.73)$ & $11(4.55)$ \\
\hline$M X$ & 64 (28.19) & $57(23.55)$ \\
\hline Unknown & $3(1.32)$ & $1(0.41)$ \\
\hline \multicolumn{3}{|l|}{ Stage } \\
\hline$|\&| \mid$ & $174(76.65)$ & $187(77.27)$ \\
\hline III \& IV & $50(22.03)$ & $51(21.07)$ \\
\hline Unknow & $3(1.32)$ & $4(1.66)$ \\
\hline
\end{tabular}

(48.4\%) and wild-type TP53 $\left(\right.$ TP5 $\left.3^{\mathrm{w}}\right)$ in 242 cases (51.6\%). All patients with and without TP5 $3^{\text {mut }}$ were included; Table 1 lists their clinical characteristics. The TP5 $3^{\text {mut }}$ patients, i.e., 105 men (46.26\%) and 122 women (53.74\%), were aged $33-87$ years (mean age, $65.4 \pm 10.20$ years). Based on the
AJCC cancer classification, 174 cases $(76.65 \%)$ had stage I and II disease, 50 cases (22.03\%) had stage III and IV disease, and 3 cases $(1.32 \%)$ had disease of unknown stage. Among the TP $53^{\mathrm{wt}}$ patients, there were 109 men (45.04\%) and 133 women $(54.96 \%$ ), aged $39-88$ years (mean age, $66.68 \pm 9.62$ years). There were 187 cases $(77.27 \%)$ with stage I and II disease, 51 cases $(21.07 \%)$ with stage III and IV disease, and 4 cases (1.66\%) with disease of unknown stage.

\section{Somatic mutational landscape of LUAD with and without TP5 $3^{\text {mut }}$}

All mutations of each gene in each sample were counted and analyzed. The overall mutational landscape is schematically represented in a waterfall plot (Figure 1), in which we identified that $100 \%$ of TP53 mutants contained diverse mutation types, while only $86.5 \%$ of the $T P 53^{\text {wt }}$ cases had mutations. The general information of the mutations in $T P 53^{\mathrm{mut}}$ and TP5 $3^{\mathrm{wt}}$ cases were shown in Figure S2, and the mutations were classified into groups, where missense mutation comprised the largest fraction (Figure S2A,S2G), single-nucleotide polymorphism (SNP) occurred more frequently than deletions or insertions (Figure S2B, S2H), and $\mathrm{C}>\mathrm{A}$ was the most common single-nucleotide variant (SNV) in TP5 $3^{\mathrm{mut}}$ and TP5 $3^{\mathrm{wt}}$ LUAD (Figure S2C,S2I). Furthermore, altered bases in each sample were calculated and showed in box plots (Figure S2D,S2E,S2J,S2K). The top 10 most frequently mutated signatures of LUAD were showed in horizontal histogram with percentage as follows (from high to low): TP53 $3^{\text {mut }}$ (Figure S2F): TP53 (100\%), TTN (56\%), MUC16 (51\%), RYR2 (48\%), CSMD3 (46\%), ZFHX4 (39\%), LRP1B (38\%), USH2A (37\%), XIRP2 (31\%), and FLG (30\%); while that for TP5 $3^{\mathrm{wt}}$ was: TTN $(31 \%)$, KRAS (30\%), MUC16 (28\%), CSMD3 (24\%), LRP1B (22\%), RYR2 (20\%), KEAP1 (19\%), USH2A (18\%), ZFHX4 (17\%), and STK11 (16\%) (Figure S2L). Figure S3 shows the coincident and exclusive associations across the mutated genes.

\section{TMB comparison and correlation to survival outcome}

The TMB value in each case was calculated, and the patients with TP $53^{\text {mut }}$ had higher TMB level than the $T P 53^{\mathrm{wt}}$ patients; corresponding results are shown in a box plot $(\mathrm{P}<0.001 ;$ Figure $2 A)$. Then, we divided the patients into high- and low-TMB groups as described in the method, and analyzed the prognostic significance of TMB 


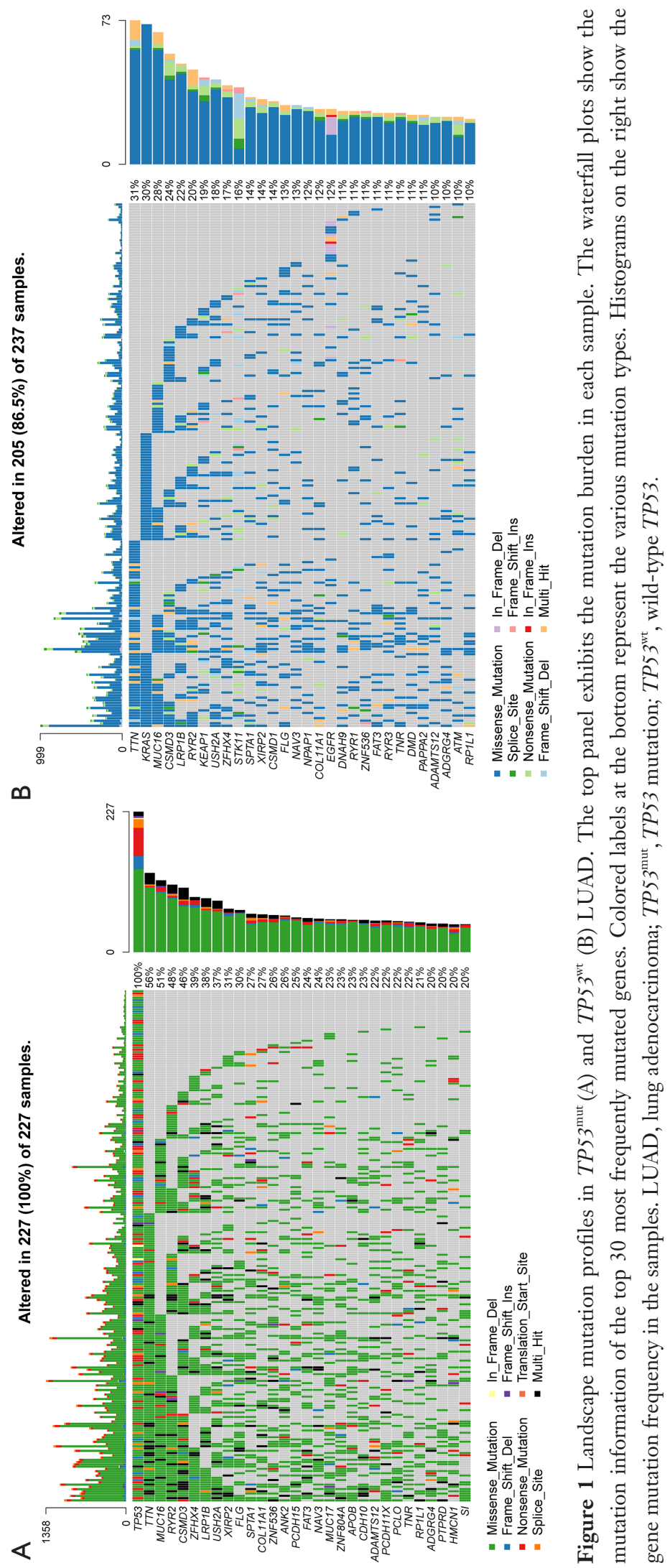




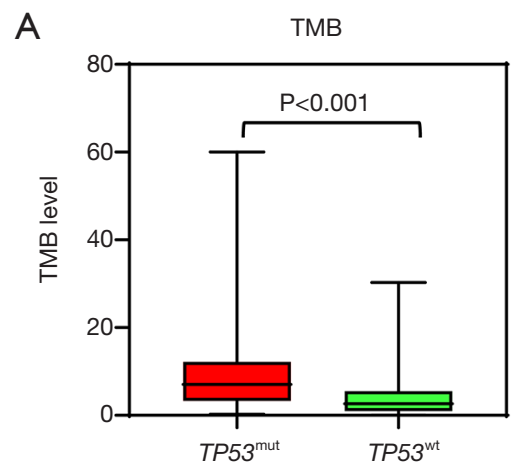

B

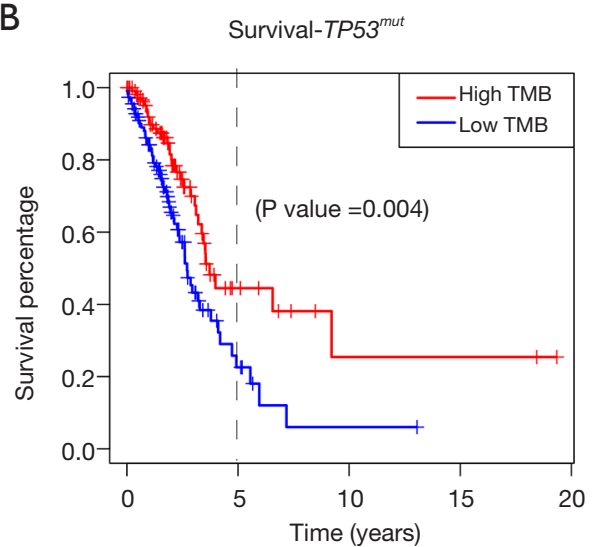

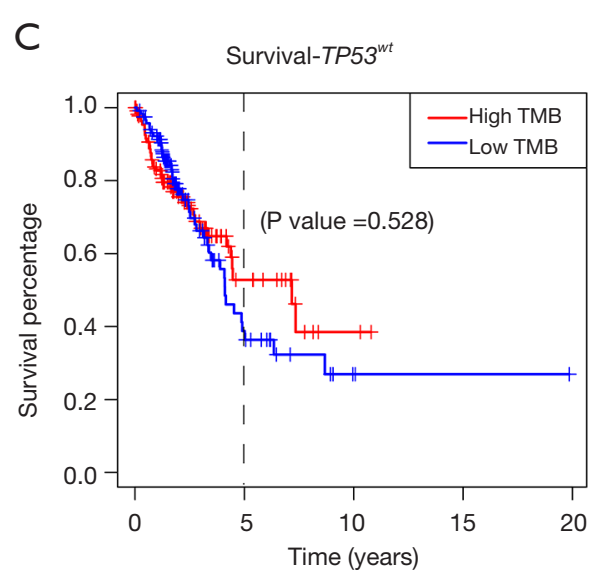

Figure 2 Prognostic analysis of TMB levels in TP5 $3^{\text {mut }}$ and TP5 $3^{\mathrm{wt}}$ LUAD. (A) TP53 mutants had higher TMB values than wild-type TP53 ( $\mathrm{P}<0.001)$. (B) Correlation of TMB levels with survival outcomes in TP5 $3^{\text {mut }}$ LUAD. (C) Correlation of TMB levels with survival outcomes in TP5 $3^{\text {wt }}$ LUAD. Red and blue curves represent the high-TMB and low-TMB groups, respectively. The dotted lines show the 5 -year survival rates. $\mathrm{P}<0.05$ indicates statistical significance. TMB, tumor mutation burden; LUAD, lung adenocarcinoma; TP53 ${ }^{\text {mut }}$, TP 53 mutation; TP5 $3^{\mathrm{wt}}$, wild-type TP53.

for overall survival (OS) separately. TP5 $3^{\text {mut }}$ patients with the low-TMB levels had significantly shorter OS than those with the high-TMB levels $(\mathrm{P}=0.004$; Figure $2 B)$. As a comparison, there was no significance in OS for TP5 $3^{\mathrm{wt}}$ patients between the high-TMB and low-TMB groups $(\mathrm{P}=0.528$, Figure $2 C)$. These findings indicate that the TMB value has a prognostic role in LUAD, especially in TP5 $3^{\text {mut }}$ patients. In addition, we examined the association between TMB levels and the patients' clinical characteristics. Higher TMB value correlated with age $(\mathrm{P}=0.005$, Figure $\mathrm{S} 4 \mathrm{~A})$ and male gender $(\mathrm{P}=0.008$, Figure $\mathrm{S} 4 \mathrm{~B})$. However, no significant relationship of TMB level was observed in the AJCC-T stage, AJCC-N stage, AJCC-M stage, and AJCC-stage I-IV groups (Figure S4C-S4F).

\section{Relationship of PD-1/PD-L1 expression with TMB value and survival outcome}

PD-1 and PD-L1 are well-known ICB targets in LUAD. PD-1 and PD-L1-directed tumor immunotherapy has become more widely used for cancer in clinical practice. We explored the relationship of PD-1/PD-L1 expression levels with TMB value and found that there was no significant relevance of PD-1/PD-L1 expression to TMB levels (Figure $3 A, B$ ). Prognosis analysis revealed no significance for OS in TP $53^{\text {mut }}$ patients grouped according to PD-1/ PD-L1 differential expression levels (Figure 3C). These indicated that PD-1/PD-L1 expression levels were not the suitable prognostic indicators for TP5 $3^{\text {mut }}$ patients. 

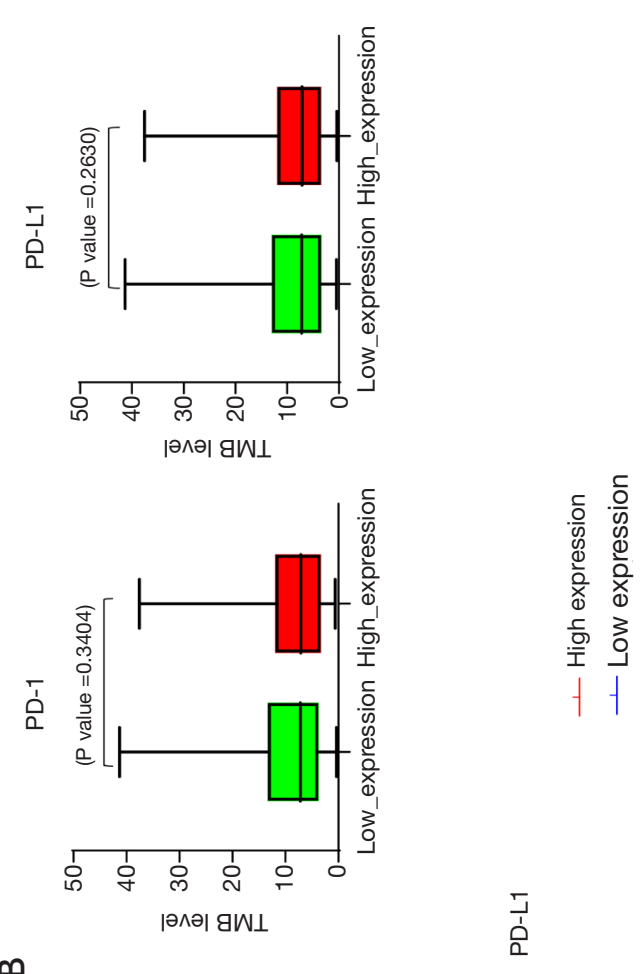

$\infty$
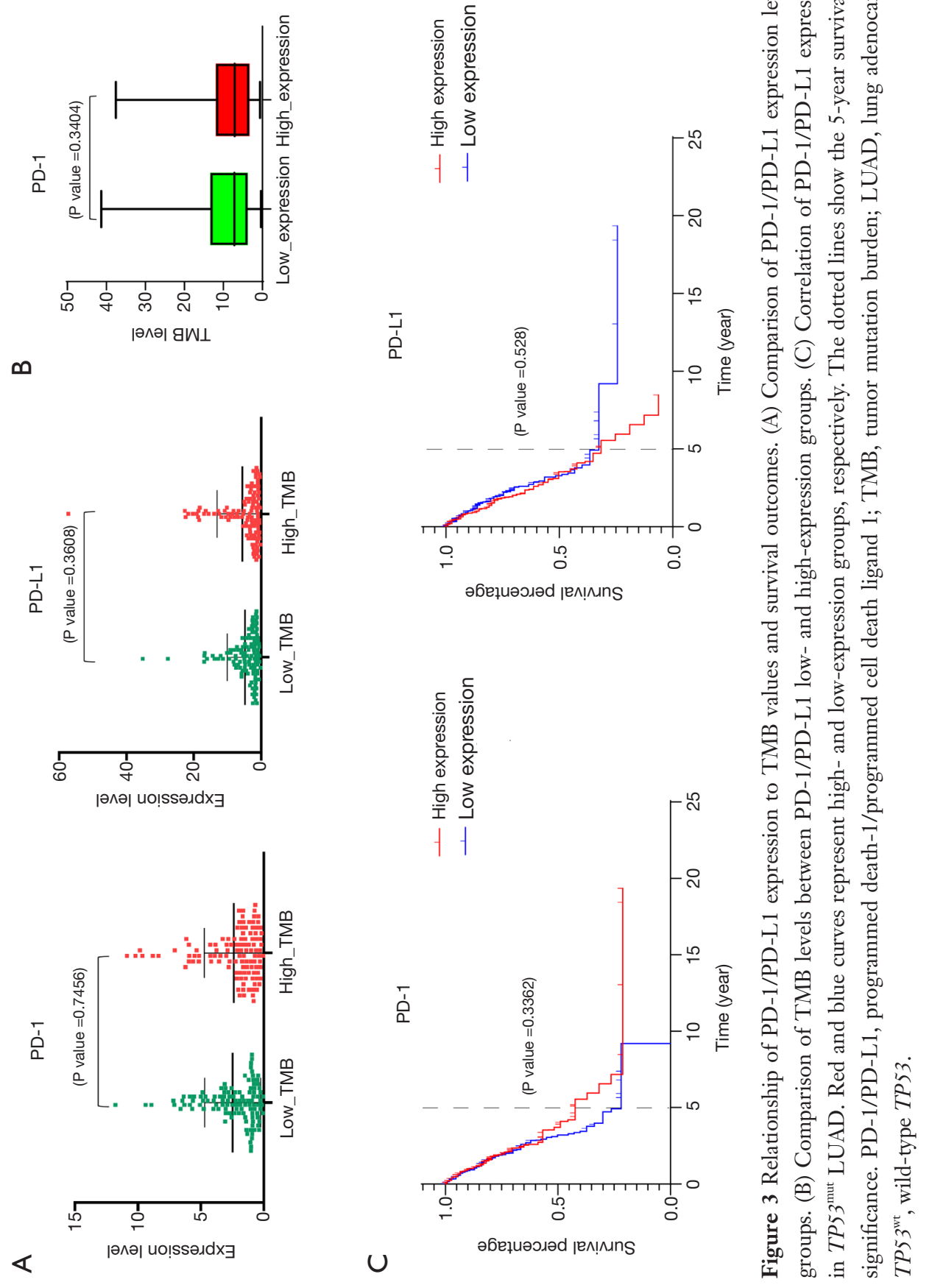
Comparison analysis of gene expression between TMB groups in TP5 $3^{\text {mut }}$ LUAD

As shown in Figure 4A, the genome expression levels in the high-TMB group were typically decreased compared to that in the low-TMB group. Differential analysis revealed 121 DEGs with $\mid \log 2 \mathrm{FCl}>1$ and $\mathrm{P}<0.05$ (https://cdn. amegroups.cn/static/public/tcr-21-565-1.xlsx). KEGG pathway analysis suggested that the enrichment of TMBrelated signatures mainly correlated with immunoinflammatory responses (Figure $4 B$, https://cdn.amegroups. $\mathrm{cn} /$ static/public/tcr-21-565-2.xlsx). GO enrichment analysis showed that the DEGs functioned mainly in cytokine activity, chemokine activity, and immune related crosstalk (Figure 4C; https://cdn.amegroups.cn/static/public/tcr21-565-3.xlsx). Furthermore, we also obtained the GSEA results for the top items, revealing that the active signaling pathways of high-TMB groups were mainly enriched in insulin, Notch, ERBB, and mTOR signaling pathways (FDR q-value $<0.25$ ) (Figure 4D). In the low-TMB group, the active pathways were often associated with Nod-like receptor, chemokine, JAK-STAT, Fc epsilon RI, Toll-like receptor, RIG-I-like receptor, B cell receptor, and PPAR signaling pathways (Figure 4D). All these findings suggested that TMB is a specific indicator for human immunity and closely related to the prognosis of $T P 53^{\mathrm{mut}}$ patients.

\section{Abundance distribution of immune cells between TMB groups in TP5 $3^{\text {mut }}$ LUAD}

We then calculated the particular proportions of 22 immune cells in each TP $53^{\text {mut }}$ sample by CIBERSORT algorithm, and showed the result in a box plot (Figure 5A). The Wilcoxon rank-sum test revealed higher infiltrating levels of CD8 T cells, plasma cells (effector B cells) and helper follicular $\mathrm{T}$ cells in the high-TMB group than that in the low-TMB group, while resting memory CD4 T cells, monocytes, resting dendritic cells, activated dendritic cells, and resting mast cells showed the opposite trend $(\mathrm{P}<0.05$; Figure 5B).

\section{Low B cell and high dendritic cell infiltrates were a risk factor and predicted poor survival outcome}

To investigate the underlying prognostic roles of various immune cells for $T P 53^{\mathrm{mut}}$ patients, we conducted univariate analysis of these signatures associated with OS. Lower infiltration of effector $B$ cells and higher infiltration of activated dendritic cells and memory B cells were correlated with poor survival outcome, while other immune infiltrates had no significant impact on OS (Figure 6). Effector B cells and activated dendritic cells were regarded as TMB-related immune infiltrates and their detection could be combined with the TMB value for predicting prognosis in TP $53^{\mathrm{mut}}$ patients.

\section{Identification of hub TMB-related genes and relationship of CNVs with immune infiltrates}

Survival risk analysis of 121 DEGs $(\mathrm{P}<0.05)$ revealed three TMB-related hub genes associated with survival outcome: FBXO36 (F-box protein 36), KLHL35 (kelch-like family member 35), and LINC00524 (long noncoding RNA, lncRNA). FBXO36 and KLHL35 expression levels correlated positively with good survival outcome, while LINC00524 was negatively correlated (Figure 7A). Furthermore, the relationship of expression levels and $\mathrm{CNVs}$ of the two hub protein-coding genes with immune cell infiltration in the LUAD microenvironment was analyzed. Partial correlation analysis revealed a positive linear association between FBXO36 expression level and CD8 $\mathrm{T}$ cell infiltrates $(\mathrm{P}<0.01$; Figure $7 B$ ), while $K L H L 35$ expression was related negatively with CD8 $\mathrm{T}$ cell and neutrophil infiltrates $(\mathrm{P}<0.01$; Figure $7 B$ ). Moreover, the immune infiltration levels compared with the samples carrying normal copy numbers of the signatures, the diverse forms of CNVs in the two hub genes commonly inhibited CD8 T cell, CD4 T cell, neutrophil, dendritic cell, macrophage, and B cell infiltrates (Figure 7C).

\section{Construction and assessment of TMBPI for TP $53^{m u t}$ LUAD}

As the vital immune signatures identified in our study were closely related to prognosis of TP5 $3^{\text {mut }}$ patients, we constructed a TMBPI through the multivariate Cox regression analysis to evaluated the predictive accuracy of the three hub TMB-related genes reported earlier. The design formulas for the TMBPI was as follows: TMBPI $=-1.602609 \times F B X O 36+-0.236524 \times K L H L 35+0.122749$ $\times$ LINC00524. Then, we divided TP5 $3^{\text {mut }}$ patients into two TMBPI levels based on the median value as the cutoff (https://cdn.amegroups.cn/static/public/tcr-21-565-4. xlsx). The ROC curve of 5 -year OS prediction was drawn to assess the predictive accuracy, with area under the curve $(\mathrm{AUC})=0.674$ (Figure 7D). Kaplan-Meier analysis showed 


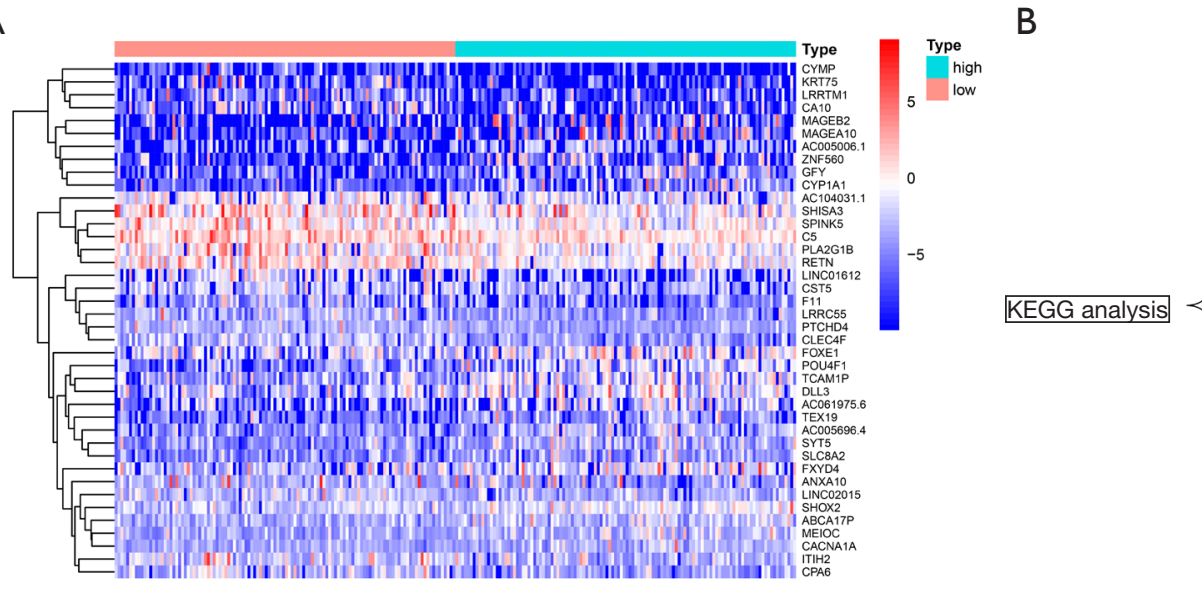

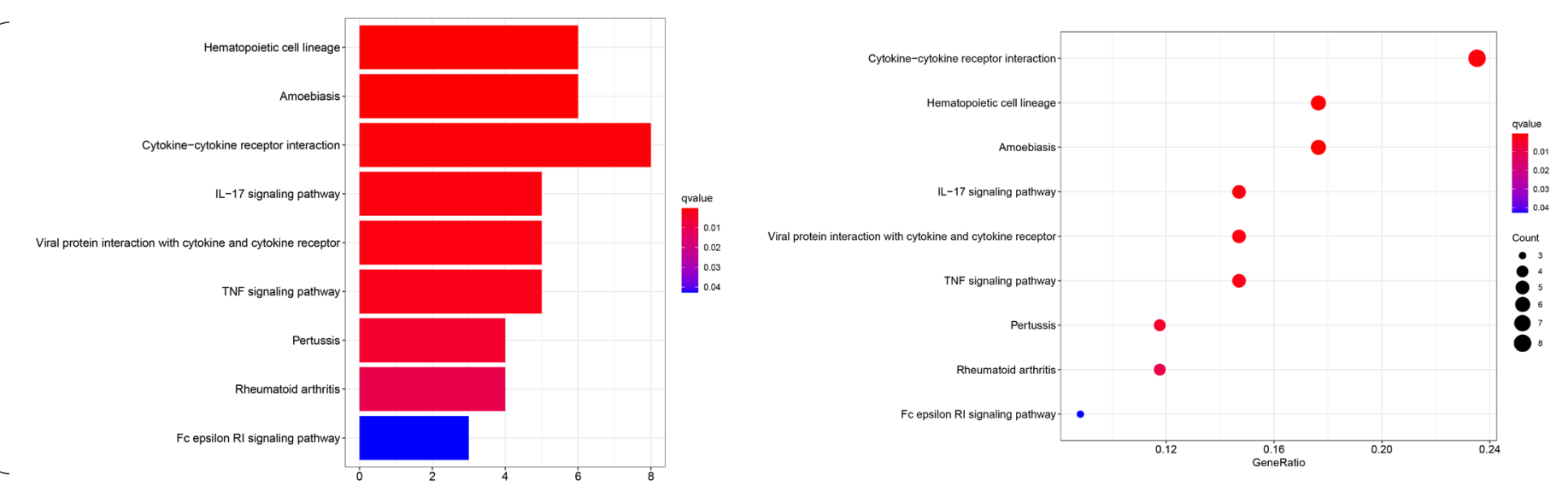
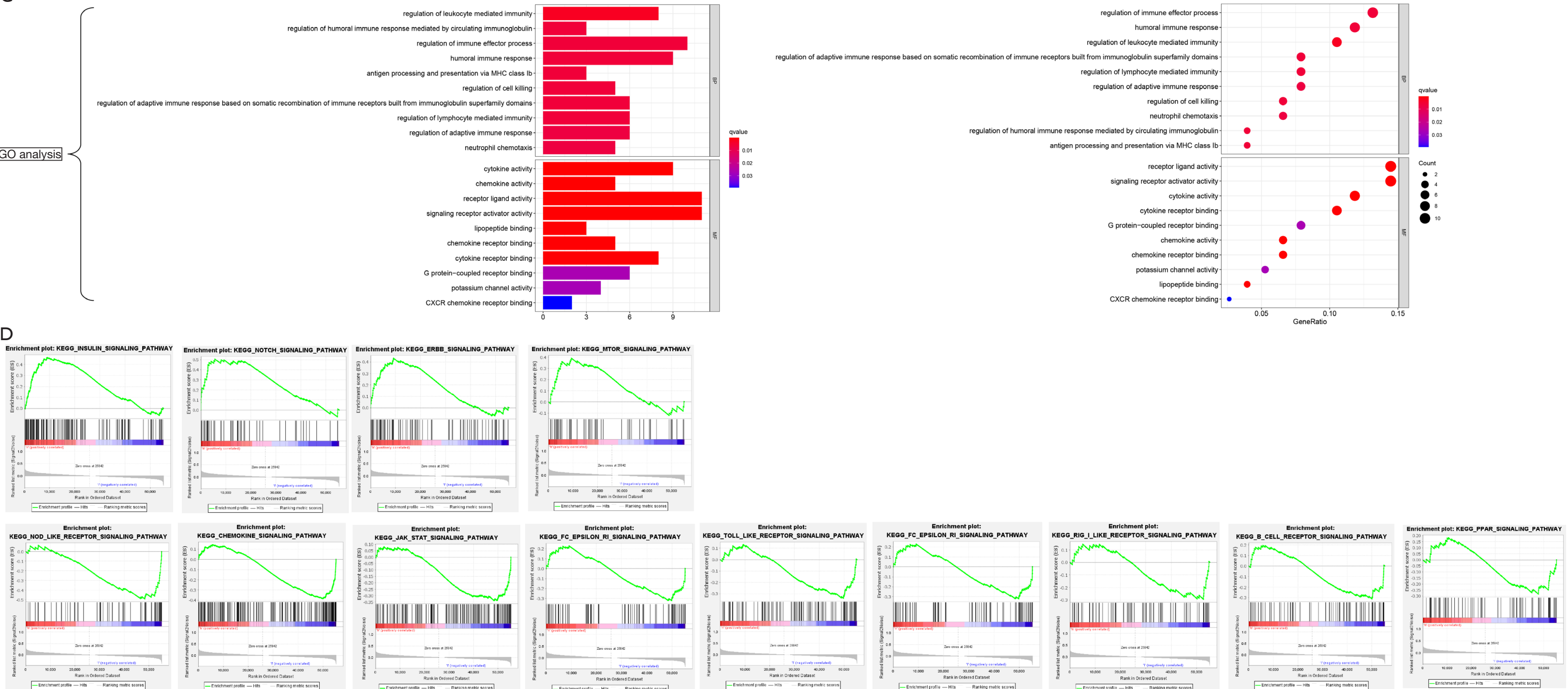

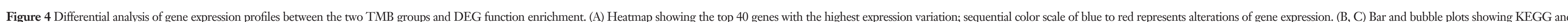

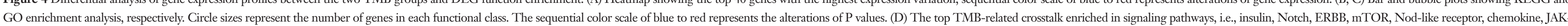

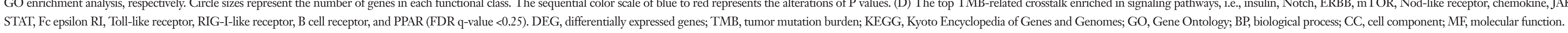



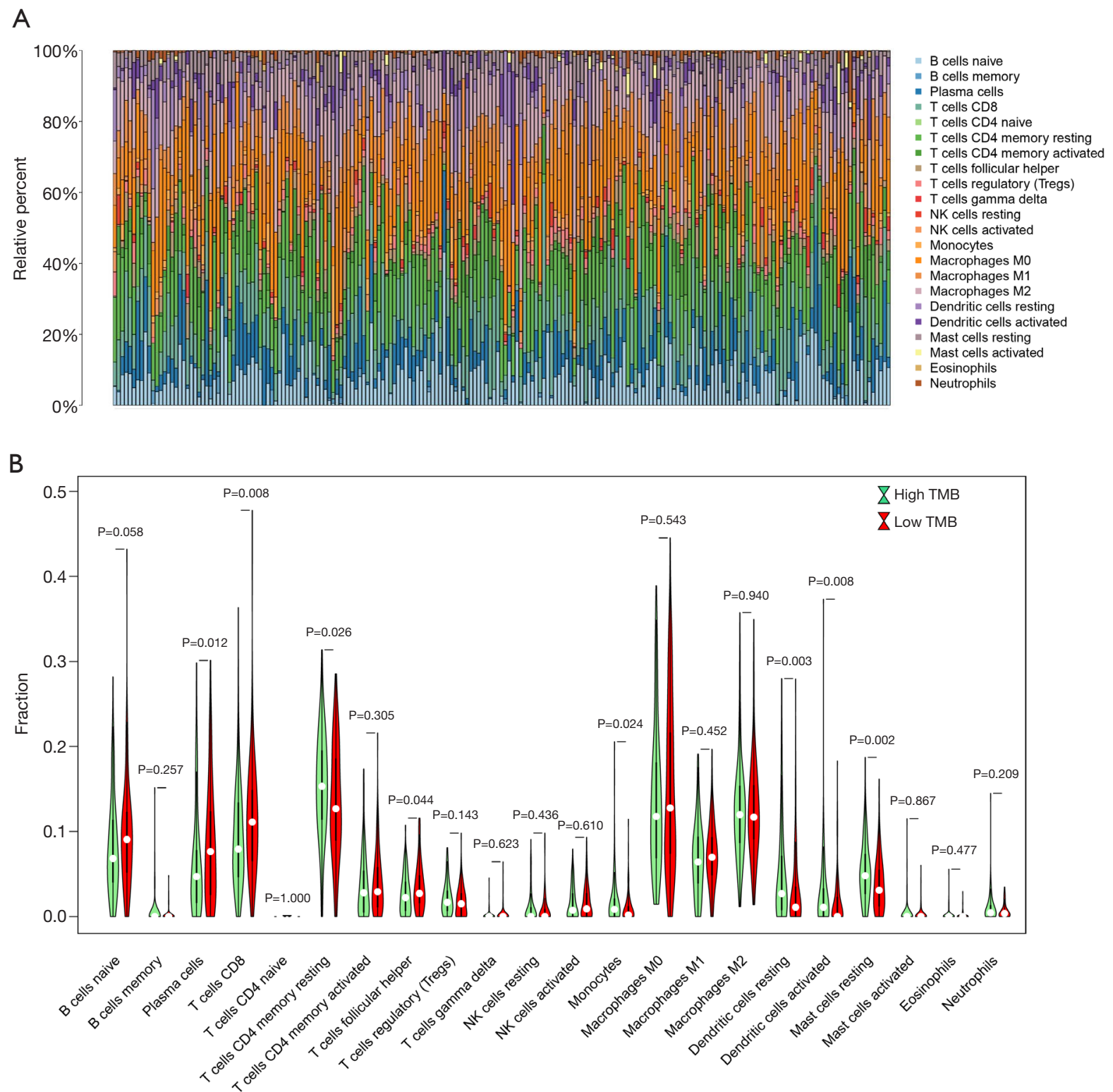

Figure 5 Comparisons of 22 important immune fractions between the two TMB groups. (A) Bar plot showing the fractions of 22 specific immune cells in each sample. (B) Violin plot showing the relationship of TMB levels to the fractions of the 22 immune cells. Green and red represent low and high TMB levels, respectively. $\mathrm{P}<0.05$ indicates statistical significance. TMB, tumor mutation burden. 

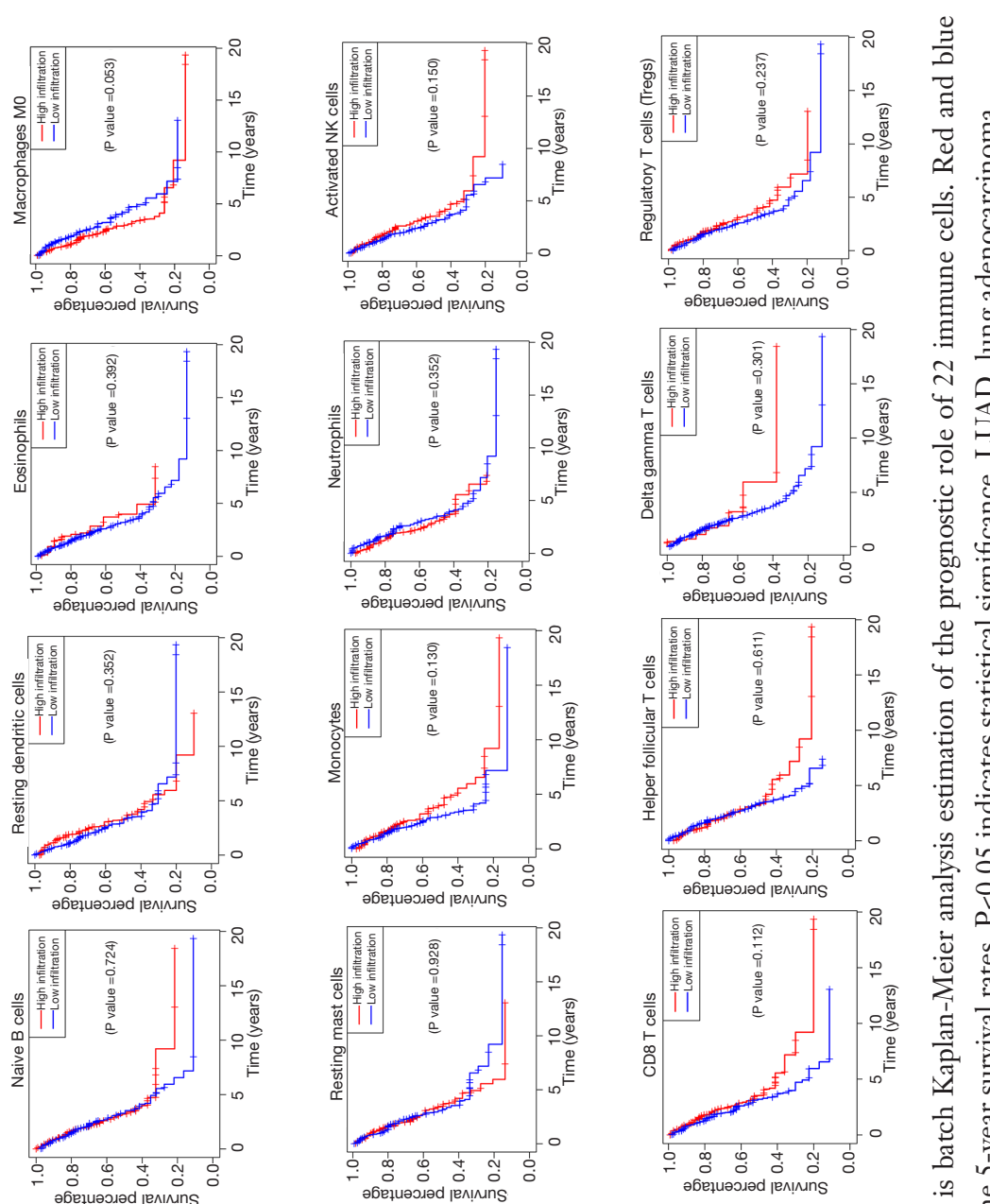

U.
0
0
0
0
0
0
0
0
0
0
0
0
0
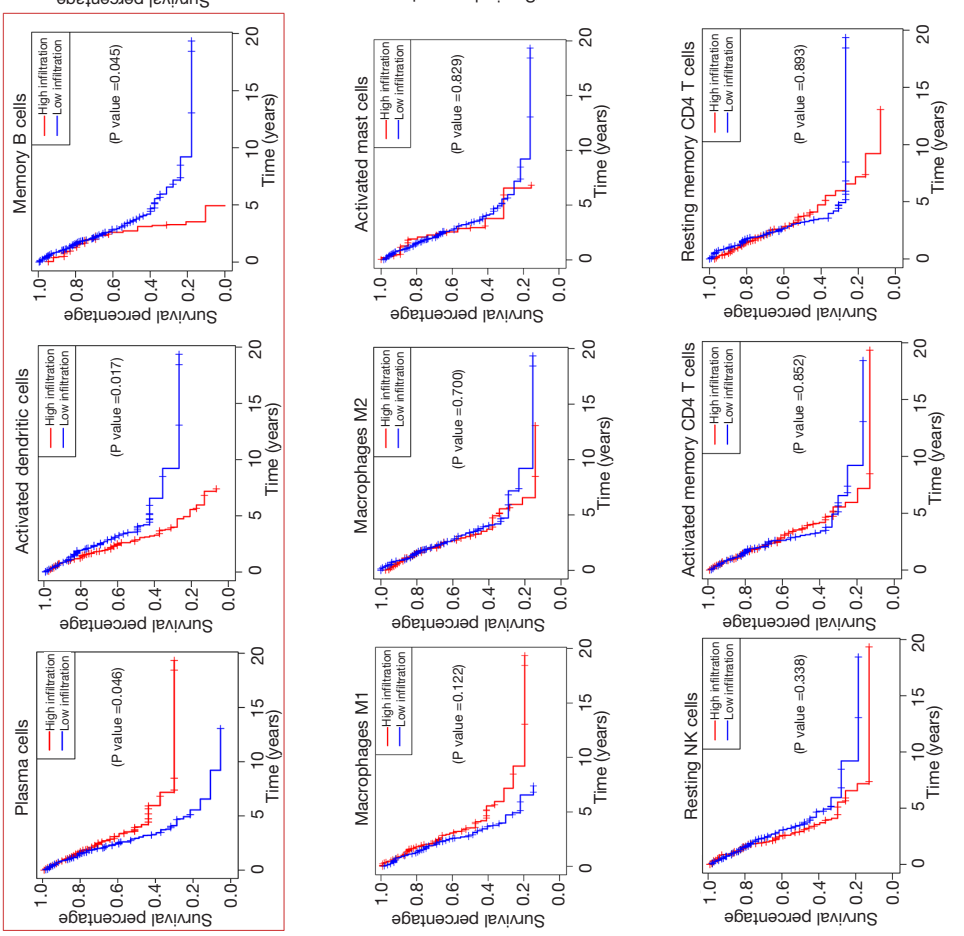

壱

ธี

.

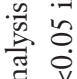

든

:

I

要

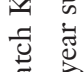

.

药

के

安密

,

竎

î.
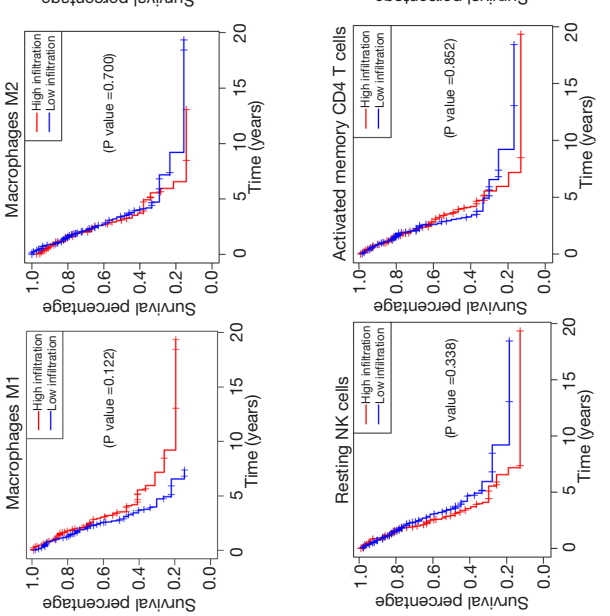

$\exists \dot{9}$

क

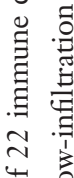

일

雍

节 $\frac{1}{60}$

荧

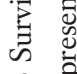

6 वे

$\underbrace{0}_{0}$ 

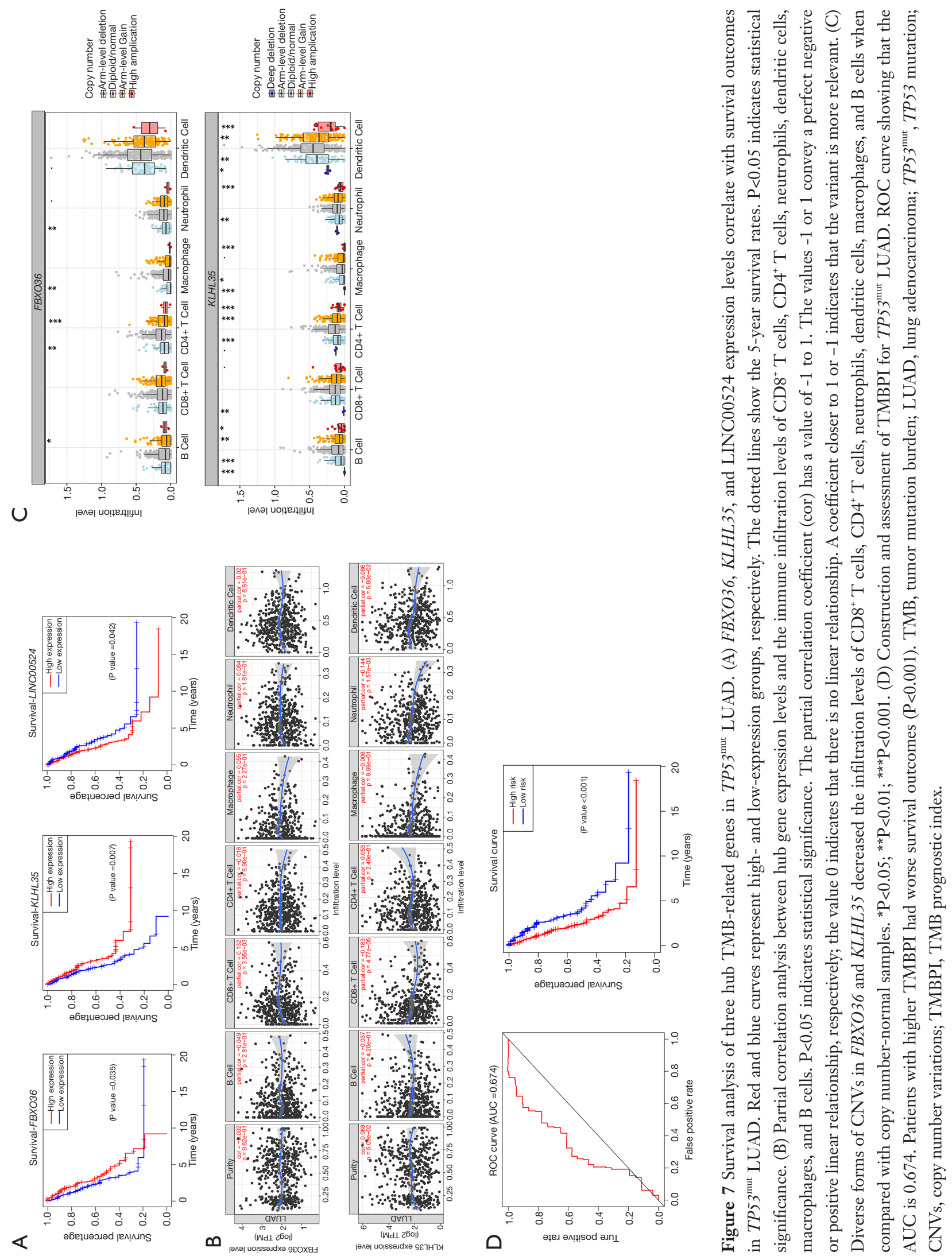
that patients with higher TMBPI had worse survival outcomes, which warrants further investigation and larger samples for validation (Figure 7D).

\section{Discussion}

TP53 is an ancient tumor suppressor gene identified in 1990 (3). Recently, there was a research boom for TP53 mutations due to its close correlation with sensitivity to targeted drug therapy and immunotherapy in lung cancer. Unlike good sensitivity to immunotherapy, TP53 mutations can lead to poor response to chemoradiotherapy and targeted drug therapy, including EGFR/ALK-TKI as the standard first-line therapy for advanced LUAD with EGFR/ALK mutations $(31,32)$. Therefore, immunotherapy currently appears to be the available effective treatment for TP $53^{\text {mut }}$ patients. However, the real-world data shows that only a fraction of TP53 mutants can obtain persistent responses and favorable long-term outcomes form immunotherapy $(11,12)$. TP53 mutation is far from an ideal independent predictor for the efficacy of immunotherapy. Therefore, effective biomarkers combined with detection of TP53 mutation for precise prediction of the efficacy of immunotherapy require further exploration.

The identification of potential biomarkers not only can screen out responders for immunotherapy, but also avoid unnecessary costs and severe toxicities for non-responders. PD-L1, TMB, and TILs, novel biomarkers for predicting immune responses, have demonstrated their efficacy in lung cancer $(13,15,17,33)$. Nonetheless, few relevant researches have focused on the association of PD-L1, TMB, and TILs with their prognostic roles in TP5 $3^{\text {mut }}$ LUAD.

In the present study, landscape analysis of genomic alterations in our cohort revealed that SNPs occurred more frequently than deletions or insertions. Among them, the $\mathrm{C}>\mathrm{A}$ nucleotide transversion signature was the most common SNV in LUAD, which differs from other cancers, including renal clear cell carcinoma and cutaneous melanoma, where the $\mathrm{C}>\mathrm{T}$ nucleotide transition signature is dominant $(30,34)$. This could be attributed to the long-term exposure to tobacco smoke in patients with LUAD (35). Moreover, the top mutated signature in TP $53^{\text {mut }}$ LUAD was TP53 (100\%), but was KRAS $(30 \%)$ in TP5 $3^{\mathrm{wt}}$ patients, and TP5 3 mutation was exclusive to KRAS mutation, with different significance. As previous reports have stated that LUAD with TP53 or KRAS mutation exhibits better immunotherapy response (7), we believe that different immune responders have specific genetic backgrounds, implying that the mechanisms of patient response to immunotherapy are also diverse.

It is well-known that TP53 gene mutations are involved in the dysfunction of DNA repair, cell growth, and apoptosis, which may lead to higher TMB in TP $53^{\text {mut }}$ tumors $(23,24)$. In the present research, we examined the TMB status in TP5 $3^{\text {mut }}$ LUAD. We discovered that $100 \%$ of $T P 53^{\mathrm{mut}}$ patients have diverse mutation types, while only $86.5 \%$ of $T P 53^{\mathrm{wt}}$ patients had mutations. This agrees with the result in Figure $2 A$, where TP5 $3^{\text {mut }}$ patients have higher TMB than TP $53^{\mathrm{wt}}$ patients. Moreover, survival analysis of TMB values revealed that higher TMB indicated better prognosis in $T P 53^{\mathrm{mut}}$ patients, but not in $T P 53^{\mathrm{wt}}$ patients. These results all indicate that the TMB value is a specific prognostic factor for $T P 53^{\text {mut }}$ patients in LUAD and that TP53 mutants are likely treated with immunotherapy. In addition, clinicopathological characteristic-related analysis showed that TMB levels correlated positively with age and male gender, but were not related to AJCC-TNM stage. Younger and male patients tended to have higher TMB levels and better prognosis, which is opposite to the results in several clinical trials that showed that older patients tend to be more sensitive to immunotherapy $(36,37)$. The potential explanations for these findings in our article needs further research. Beyond that, we found that PD-1/PD-L1 expression was not related to TMB levels and the prognosis of TP $53^{\mathrm{mut}}$ LUAD, which suggested that PD-1/PD-L1 expression is not a suitable diagnostic biomarker for $T P 53^{\mathrm{mut}}$ LUAD.

Subsequently, we conducted comparative analysis of public gene expression data between the high-TMB and low-TMB groups in TP $53^{\text {mut }}$ LUAD. Multiple DEGs functions were enriched in pathways involving immunity regulation and response, suggesting that TMB is a specific indicator for human immunity and closely related to the prognosis of TP $53^{\text {mut }}$ patients. Moreover, the differential abundance of 22 immune cells between the two TMB groups showed that high TMB had a significant impact on CD8 T cell and effector B cell enrichment, while resting memory $\mathrm{CD} 4 \mathrm{~T}$ cells, monocytes, resting dendritic cells, activated dendritic cells, and resting mast cells were abundant in the low-TMB group. As infiltration by B cells and activated dendritic cells was significantly related to survival $(\mathrm{P}<0.05)$, their alterations in TIME may be responsible for the marked differences in prognosis between the two TMB groups. The results imply that TMB levels with effector B cell and activated dendritic cell infiltrates is a potential biomarker for the prognosis of $T P 53^{\text {mut }} \mathrm{LUAD}$. 
At present, high cost and complicated technology are needed for detecting TMB and immune cell infiltration (38-40), and we attempted to build a prognostic model with hub TMB-related genes to optimize the detection. We identified three hub TMB-related genes from 121 DEGs. The diverse forms of their CNVs and their expression levels typically affected the immune infiltrates. The TMBPI prognostic model was constructed using three hub TMB-related genes for predicting prognosis in TP $53^{\text {mut }}$ LUAD, and patients with higher TMBPI had worse survival outcomes. The AUC of this predictive model was 0.674 , and further large-scale researches are required for verification and modification before clinical application.

In our study, we not only identified a special prognostic biomarker and constructed a prognostic model for TP $53^{\text {mut }}$ LUAD, but also provided some new insights for better understanding of poor prognosis of tumor patients: (I) TMB value was only associated with prognosis of patients with TP5 $3^{\text {mut }}$ LUAD, suggesting that TMB might be better to predict prognosis with coexisting factors of DNA damage repair disorder and genome instability, etc. (II) Unlike other reports that CD8 $\mathrm{T}$ cell was closely related to prognosis and immunotherapy, our study revealed effector B cell have important prognostic indicating role in $T P 53^{\mathrm{mut}}$ patients, implying that immune regulatory mechanisms were various in tumors with different genotypes. (III) PD-1/PD-L1 expression is an important biomarker for responses of patients to ICB therapy based on the most clinical trials, but it was not an appropriate prognostic predictor for TP $53^{\text {mut }}$ patients though TP53 mutation was reported closely related to human immunity. Beyond that, our findings also provided important reference for possible intervention therapy for $T P 53^{\text {mut }}$ patients with poor prognosis: activation of immunity to increase the infiltration of effector B cells in TIME.

However, our study also has limitations that should not be disregarded: (I) the association between hub TMBrelated genes and immune infiltrates in TIME lacks further verification experiments; (II) the prognostic role of TMB and its potential correlation with immune infiltrates lacks confirmation via a large clinical sample. Clinically relevant variants and large-sample trials are needed in the future.

In summary, higher TMB levels with effector B cell and activated dendritic cell infiltrates is a potential biomarker of good prognosis in TP $53^{\text {mut }}$ LUAD. In addition, the prognostic predictive model we constructed indicates that higher TMBPI predicts worse survival outcome, which warrants further validation.

\section{Acknowledgments}

We acknowledge TCGA database for providing their platforms and contributors for uploading their meaningful datasets. We would like to thank Dr. James Allen for his help in polishing our paper.

Funding: This work was supported by grants from Cancer Foundation of China (kk201400010), Henan innovative talent project (CYQ20160226, CYQ20170148).

\section{Footnote}

Reporting Checklist: The authors have completed the REMARK reporting checklist. Available at https://dx.doi. org/10.21037/tcr-21-565

Peer Review File: Available at https://dx.doi.org/10.21037/ tcr-21-565

Conflicts of Interest: All authors have completed the ICMJE uniform disclosure form (available at https://dx.doi. org/10.21037/tcr-21-565). The authors have no conflicts of interest to declare.

Ethical Statement: The authors are accountable for all aspects of the work in ensuring that questions related to the accuracy or integrity of any part of the work are appropriately investigated and resolved. The study was conducted in accordance with the Declaration of Helsinki (as revised in 2013). Institutional ethical approval and informed consent were waived.

Open Access Statement: This is an Open Access article distributed in accordance with the Creative Commons Attribution-NonCommercial-NoDerivs 4.0 International License (CC BY-NC-ND 4.0), which permits the noncommercial replication and distribution of the article with the strict proviso that no changes or edits are made and the original work is properly cited (including links to both the formal publication through the relevant DOI and the license). See: https://creativecommons.org/licenses/by-nc-nd/4.0/.

\section{References}

1. Zheng M. Classification and Pathology of Lung Cancer. Surg Oncol Clin N Am 2016;25:447-68.

2. Zhang XC, Wang J, Shao GG, et al. Comprehensive genomic and immunological characterization of Chinese 
non-small cell lung cancer patients. Nat Commun 2019;10:1772.

3. Greenblatt MS, Bennett WP, Hollstein M, et al. Mutations in the p53 tumor suppressor gene: clues to cancer etiology and molecular pathogenesis. Cancer Res 1994;54:4855-78.

4. Donehower LA, Soussi T, Korkut A, et al. Integrated Analysis of TP53 Gene and Pathway Alterations in The Cancer Genome Atlas [published correction appears in Cell Rep. 2019 Sep 10;28(11):3010]. Cell Rep 2019;28:1370-1384.e5.

5. Dong ZY, Zhong WZ, Zhang XC, et al. Potential Predictive Value of TP53 and KRAS Mutation Status for Response to PD-1 Blockade Immunotherapy in Lung Adenocarcinoma. Clin Cancer Res 2017;23:3012-24.

6. $\mathrm{Xu} \mathrm{F,} \mathrm{Lin} \mathrm{H,} \mathrm{He} \mathrm{P,} \mathrm{et} \mathrm{al.} \mathrm{A} \mathrm{TP53-associated} \mathrm{gene} \mathrm{signature}$ for prediction of prognosis and therapeutic responses in lung squamous cell carcinoma. Oncoimmunology 2020;9:1731943.

7. Li X, Xu Y, Gong F, et al. Terdependence of KRAS and TP53 mutations in predicting benefit from immune checkpoint inhibitor (ICI) in non-squamous NSCLC. Ann Oncol 2020;31:S852.

8. Vaddepally RK, Kharel P, Pandey R, et al. Review of Indications of FDA-Approved Immune Checkpoint Inhibitors per NCCN Guidelines with the Level of Evidence. Cancers (Basel) 2020;12:738.

9. Loria R, Giliberti C, Bedini A, et al. Very low intensity ultrasounds as a new strategy to improve selective delivery of nanoparticles-complexes in cancer cells. J Exp Clin Cancer Res 2019;38:1.

10. Gkolfinopoulos S, Mountzios G. Recent clinical trials of immunotherapy in non-small-cell lung cancer. Immunotherapy 2019;11:461-6.

11. DeLeon TT, Almquist DR, Kipp BR, et al. Assessment of clinical outcomes with immune checkpoint inhibitor therapy in melanoma patients with CDKN2A and TP53 pathogenic mutations. PLoS One 2020;15:e0230306.

12. Wang S, Jiang $M$, Yang $Z$, et al. The role of distinct comutation patterns with TP53 mutation in immunotherapy for NSCLC. Genes Dis 2020. In press.

13. Patel SP, Kurzrock R. PD-L1 Expression as a Predictive Biomarker in Cancer Immunotherapy. Mol Cancer Ther 2015;14:847-56.

14. Dudley JC, Lin MT, Le DT, et al. Microsatellite Instability as a Biomarker for PD-1 Blockade. Clin Cancer Res 2016;22:813-20.

15. Kandoth C, McLellan MD, Vandin F, et al. Mutational landscape and significance across 12 major cancer types.
Nature 2013;502:333-9.

16. Efremova M, Finotello F, Rieder D, et al. Neoantigens Generated by Individual Mutations and Their Role in Cancer Immunity and Immunotherapy. Front Immunol 2017;8:1679.

17. Zito Marino F, Ascierto PA, Rossi G, et al. Are tumorinfiltrating lymphocytes protagonists or background actors in patient selection for cancer immunotherapy? Expert Opin Biol Ther 2017;17:735-46.

18. Rizvi NA, Hellmann MD, Snyder A, et al. Cancer immunology. Mutational landscape determines sensitivity to PD-1 blockade in non-small cell lung cancer. Science 2015;348:124-8.

19. Mok TSK, Wu YL, Kudaba I, et al. Pembrolizumab versus chemotherapy for previously untreated, PD-L1expressing, locally advanced or metastatic non-small-cell lung cancer (KEYNOTE-042): a randomised, open-label, controlled, phase 3 trial. Lancet 2019;393:1819-30.

20. Dupuis F, Lamant L, Gerard E, et al. Clinical, histological and molecular predictors of metastatic melanoma responses to anti-PD-1 immunotherapy. Br J Cancer 2018;119:193-9.

21. Duan Q, Zhang H, Zheng J, et al. Turning Cold into Hot: Firing up the Tumor Microenvironment. Trends Cancer 2020;6:605-18.

22. Galon J, Bruni D. Approaches to treat immune hot, altered and cold tumours with combination immunotherapies. Nat Rev Drug Discov 2019;18:197-218.

23. Rivlin N, Brosh R, Oren M, et al. Mutations in the p53 Tumor Suppressor Gene: Important Milestones at the Various Steps of Tumorigenesis. Genes Cancer 2011;2:466-74.

24. Burtness B, Deneka A, Baca Y, et al. Correlation of tumor mutational burden (TMB) with CDKN2A and TP53 mutation in HPV-negative head and neck squamous cell carcinoma (HNSCC). J Clin Oncol 2020;38:6552.

25. Serra P, Petat A, Maury JM, et al. Programmed cell deathligand 1 (PD-L1) expression is associated with RAS/ TP53 mutations in lung adenocarcinoma. Lung Cancer 2018;118:62-8.

26. Xu C, Zhang ZH. Correlation between Programmed Death-1 Ligand-1 and p53 in Patients with Lung Adenocarcinoma. Chin Med J (Engl) 2018;131:990-3.

27. Horn L, Spigel DR, Vokes EE, et al. Nivolumab Versus Docetaxel in Previously Treated Patients With Advanced Non-Small-Cell Lung Cancer: Two-Year Outcomes From Two Randomized, Open-Label, Phase III Trials (CheckMate 017 and CheckMate 057). J Clin Oncol 
2017;35:3924-33.

28. Mayakonda A, Lin DC, Assenov Y, et al. Maftools: efficient and comprehensive analysis of somatic variants in cancer. Genome Res 2018;28:1747-56.

29. Li T, Fan J, Wang B, et al. TIMER: A Web Server for Comprehensive Analysis of Tumor-Infiltrating Immune Cells. Cancer Res 2017;77:e108-10.

30. Zhang C, Li Z, Qi F, et al. Exploration of the relationships between tumor mutation burden with immune infiltrates in clear cell renal cell carcinoma. Ann Transl Med 2019;7:648.

31. Hou H, Qin K, Liang Y, et al. Concurrent TP53 mutations predict poor outcomes of EGFR-TKI treatments in Chinese patients with advanced NSCLC. Cancer Manag Res 2019;11:5665-75.

32. Canale M, Petracci E, Delmonte A, et al. Impact of TP53 Mutations on Outcome in EGFR-Mutated Patients Treated with First-Line Tyrosine Kinase Inhibitors. Clin Cancer Res 2017;23:2195-202.

33. Pan JH, Zhou H, Cooper L, et al. LAYN Is a Prognostic Biomarker and Correlated With Immune Infiltrates in Gastric and Colon Cancers. Front Immunol 2019;10:6.

34. Yan J, Wu X, Yu J, et al. Prognostic Role of Tumor Mutation Burden Combined With Immune Infiltrates

Cite this article as: Fu J, Li Y, Li C, Tong Y, Li M, Cang S. A special prognostic indicator: tumor mutation burden combined with immune infiltrates in lung adenocarcinoma with TP53 mutation. Transl Cancer Res 2021;10(9):3963-3978. doi: 10.21037/ tcr-21-565 in Skin Cutaneous Melanoma Based on Multi-Omics Analysis. Front Oncol 2020;10:570654.

35. Alexandrov LB, Ju YS, Haase K, et al. Mutational signatures associated with tobacco smoking in human cancer. Science 2016;354:618-22.

36. Hamid O, Robert C, Daud A, et al. Five-year survival outcomes for patients with advanced melanoma treated with pembrolizumab in KEYNOTE-001. Ann Oncol 2019;30:582-8.

37. Robert C, Ribas A, Schachter J, et al. Pembrolizumab versus ipilimumab in advanced melanoma

(KEYNOTE-006): post-hoc 5-year results from an openlabel, multicentre, randomised, controlled, phase 3 study. Lancet Oncol 2019;20:1239-51.

38. Meléndez B, Van Campenhout C, Rorive S, et al. Methods of measurement for tumor mutational burden in tumor tissue. Transl Lung Cancer Res 2018;7:661-7.

39. Saha D, Rabkin SD. Immunohistochemistry for TumorInfiltrating Immune Cells After Oncolytic Virotherapy. Methods Mol Biol 2020;2058:179-90.

40. Ino Y, Yamazaki-Itoh R, Shimada K, et al. Immune cell infiltration as an indicator of the immune microenvironment of pancreatic cancer. Br J Cancer 2013;108:914-23. 


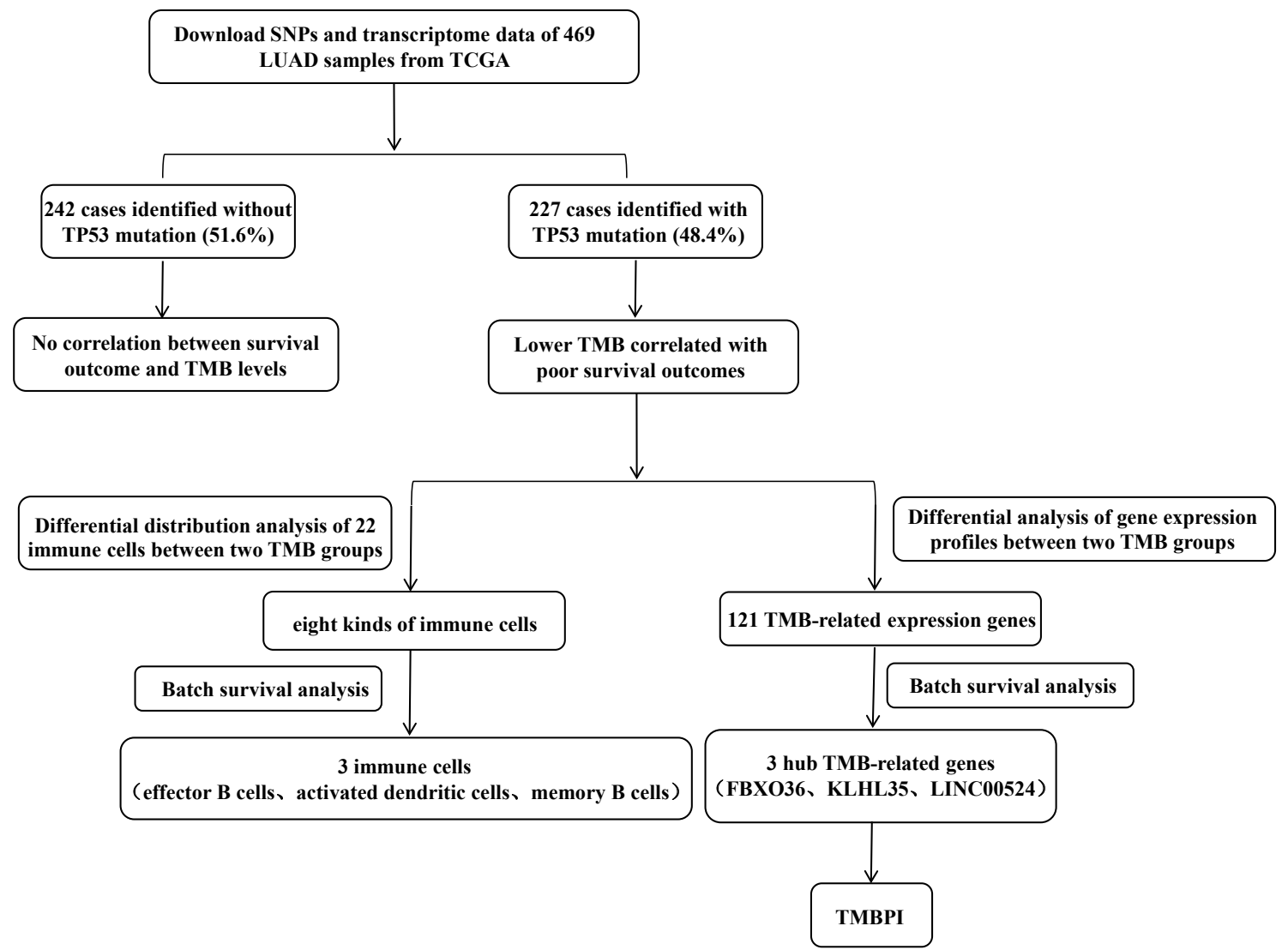

Figure S1 Workflow of this study. TMB, tumor mutation burden. TMBPI, TMB prognostic index. 


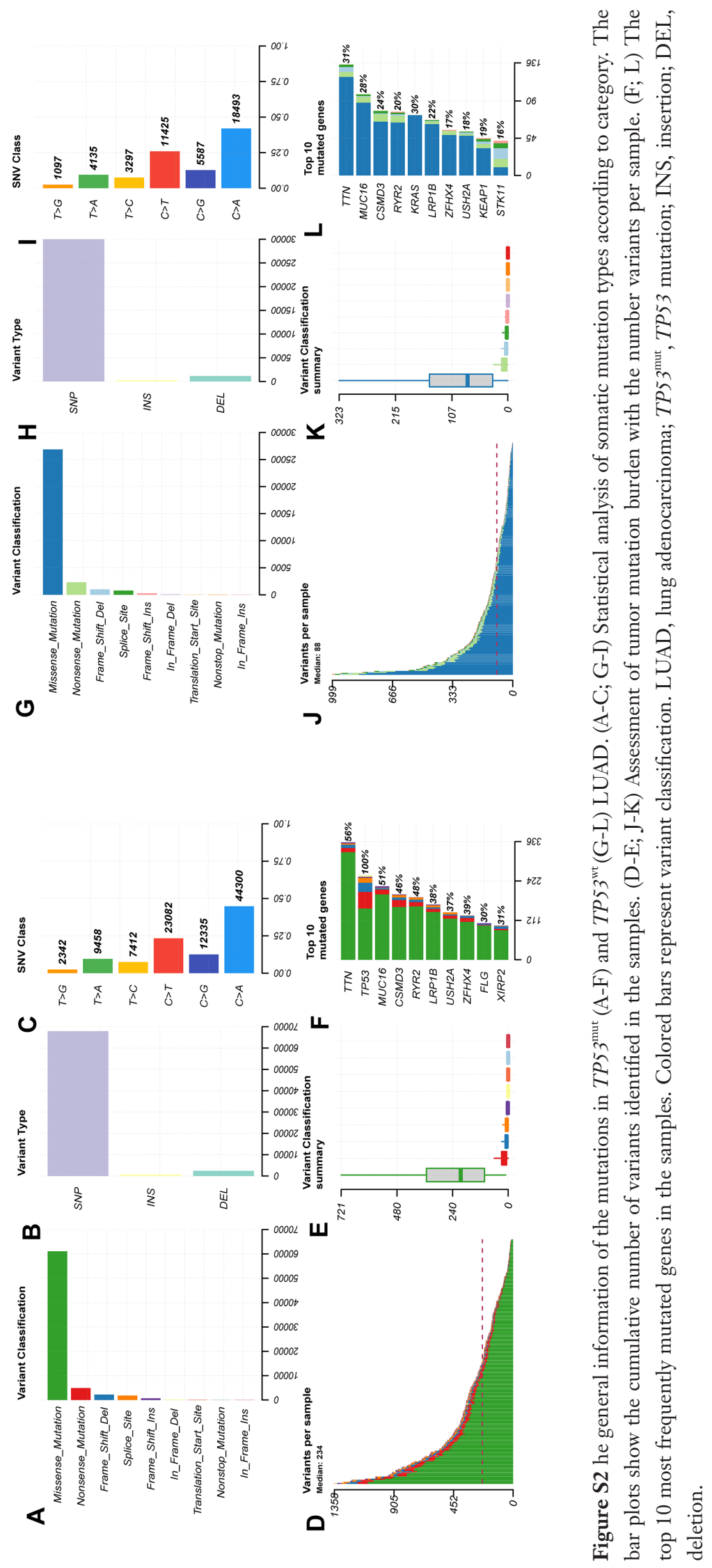

(c) Translational Cancer Research. All rights reserved. 

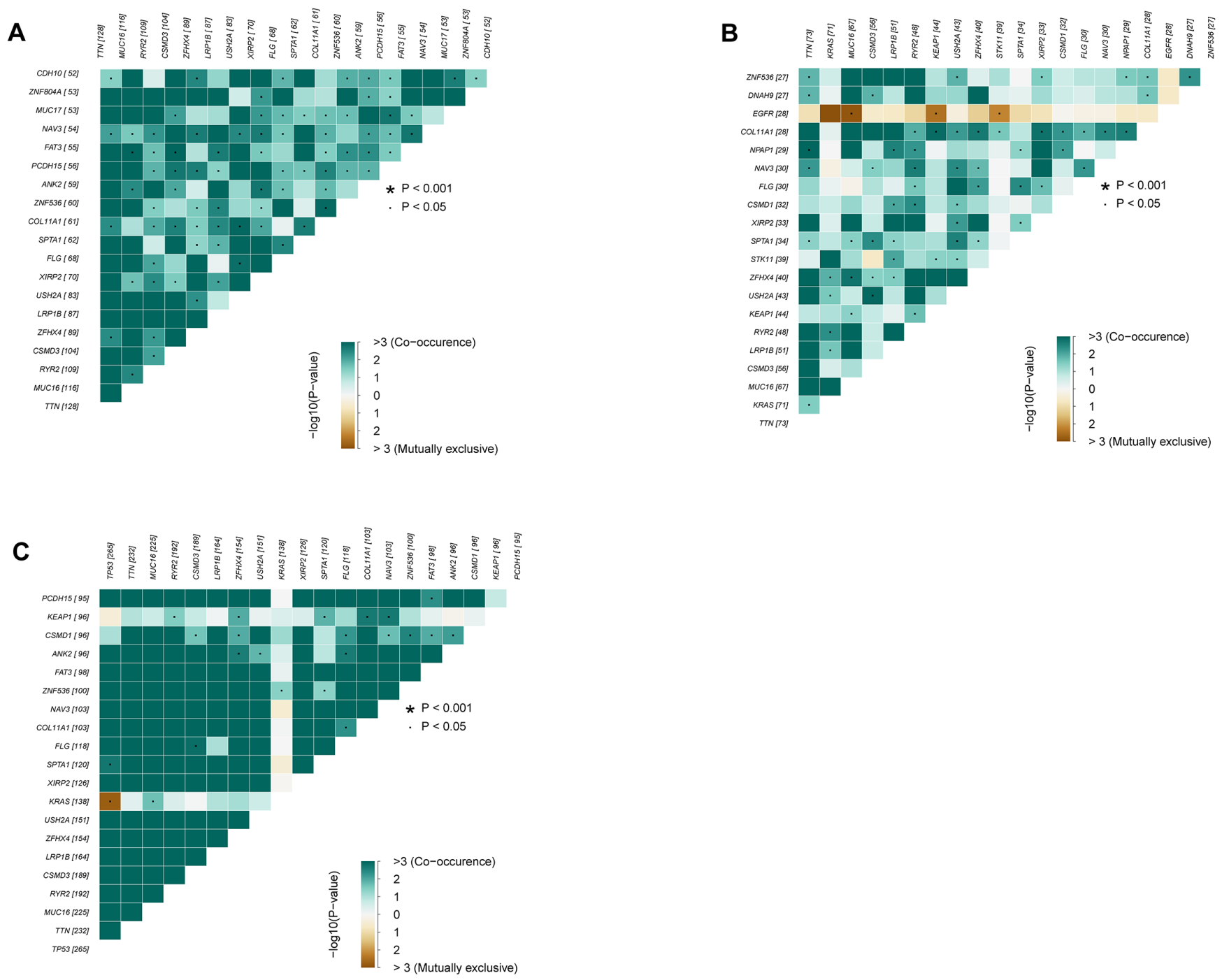

Figure S3 The coincident and exclusive associations across the mutated genes in TP5 $3^{\mathrm{mut}}(\mathrm{A})$, TP5 $3^{\mathrm{wt}}(\mathrm{B})$ and all (C) LUAD. Green represents co-occurrence and red represents mutually exclusive relationships $\left({ }^{*} \mathrm{P}<0.001 ;{ }^{\bullet} \mathrm{P}<0.05\right)$. LUAD, lung adenocarcinoma; TP $53^{\text {mut }}$, TP53 mutation; TP53 $3^{\mathrm{wt}}$, wild-type TP53. 
A

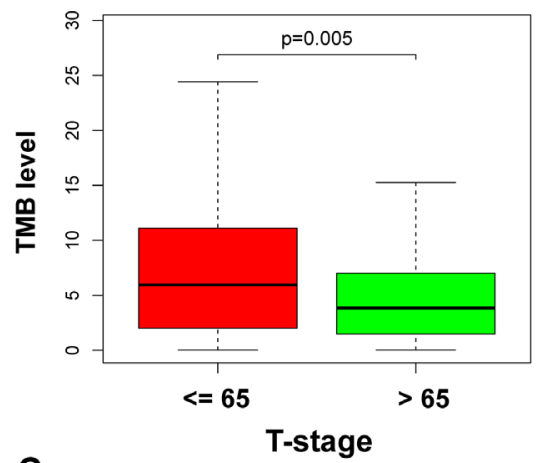

C
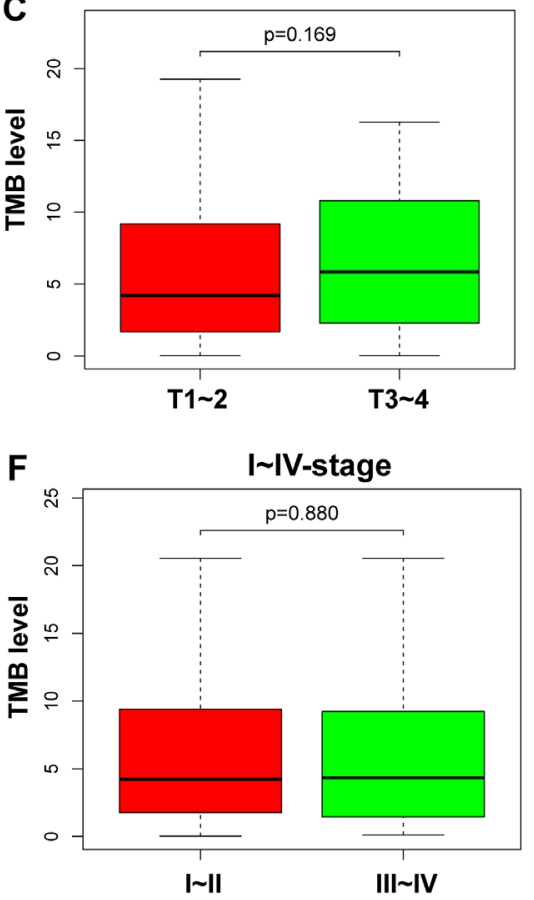

B

Gender
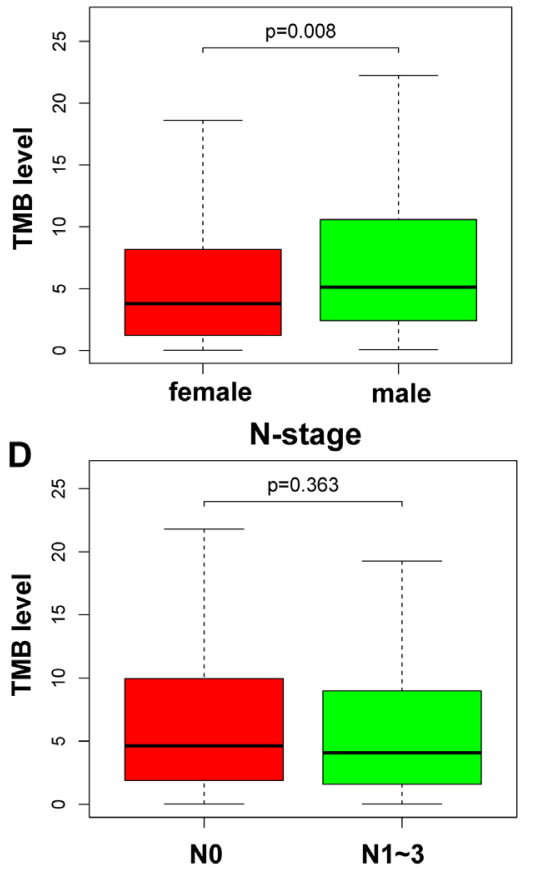

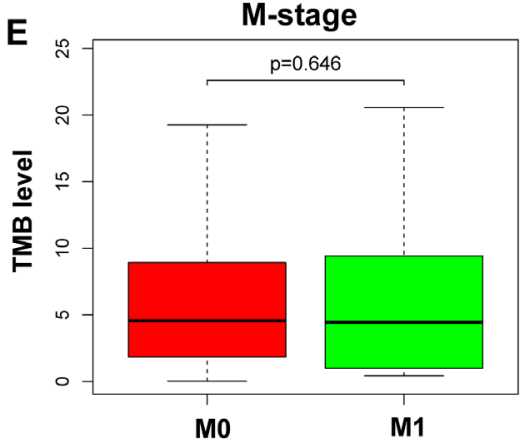

Figure S4 Correlation of TMB levels with clinical features. (A, B) Higher TMB levels related significantly to the $\leq 65$-year age group $(\mathrm{P}=0.005)$ and male gender $(\mathrm{P}=0.003)$. ( $\mathrm{C}-\mathrm{F})$ No significant difference of TMB levels was observed for AJCC-T stage, AJCC-N stage, AJCC-M stage, and AJCC-I IV stage. Red and green represent the different groups in each box plot. TMB, tumor mutation burden. 